\title{
Medium Effects in Kaon and Antikaon Production in Nuclear Collisions at Subthreshold Beam Energies
}

\author{
F. Laue $^{a}$, C. Sturm ${ }^{b}$, I. Böttcher ${ }^{d}$, M. Dębowskie ${ }^{e}$ A. Förster ${ }^{b}$, E. Grosse ${ }^{f}$, P. Koczoñ ${ }^{a}$, \\ B. Kohlmeyer ${ }^{d}$, M. Mang ${ }^{a}$, L. Naumann ${ }^{f}$, H. Oeschler ${ }^{b}$, F. Pühlhofer ${ }^{d}$, E. Schwab $^{a}$, \\ P. Senger ${ }^{a}$, Y. Shin ${ }^{c}$, J. Speer ${ }^{d}$, H. Ströbele ${ }^{c}$, G. Surowka ${ }^{a, e}$, F. Uhlig ${ }^{b}$ A. Wagner ${ }^{a, *}$, \\ W. Waluśe \\ (KaoS Collaboration) \\ ${ }^{a}$ Gesellschaft für Schwerionenforschung, D-64220 Darmstadt, Germany \\ ${ }^{b}$ Technische Universität Darmstadt, D-64289 Darmstadt, Germany \\ ${ }^{c}$ Johann Wolfgang Goethe Universität, D-60325 Frankfurt am Main, Germany \\ ${ }^{d}$ Phillips Universität, D-35037 Marburg, Germany \\ e Jagiellonian University, PL-30-059 Kraków, Poland \\ ${ }^{f}$ Forschungszentrum Rossendorf, D-01314 Dresden and Technische Universität Dresden, \\ Germany \\ ${ }^{*}$ Present address: NSCL Michigan State University, East Lansing, MI 48824-1321, USA \\ PACS numbers: 25.75.Dw
}

\begin{abstract}
Production cross sections of $\mathrm{K}^{+}$and $\mathrm{K}^{-}$mesons have been measured in $\mathrm{C}+\mathrm{C}$ collisions at beam energies per nucleon below and near the nucleon-nucleon threshold. At a given beam energy, the spectral slopes of the $\mathrm{K}^{-}$mesons are significantly steeper than the ones of the $\mathrm{K}^{+}$mesons. The excitation functions for $\mathrm{K}^{+}$and $\mathrm{K}^{-}$mesons nearly coincide when correcting for the threshold energy. In contrast, the $\mathrm{K}^{+}$yield exceeds the $\mathrm{K}^{-}$yield by a factor of about 100 in proton-proton collisions at beam energies near the respective nucleon-nucleon thresholds.
\end{abstract}


The properties of strange mesons in a medium of finite baryon density are essential for our understanding of the strong interaction. According to various theoretical approaches, antikaons feel strong attractive forces in the nuclear medium whereas the in-medium kaonnucleon potential is expected to be slightly repulsive [1 5]. Predictions have been made that the effective mass of the $\mathrm{K}^{-}$meson decreases with increasing nuclear density leading to $\mathrm{K}^{-}$ condensation in neutron stars above 3 times saturation density $\rho_{0}$. This effect is expected to influence significantly the evolution of supernova explosions: the $\mathrm{K}^{-}$condensate softens the nuclear equation of state and thus causes a core with 1.5 - 2 solar masses to collapse into a black hole rather than to form a neutron star [6.7].

Experimental evidence for the attractive in-medium $\mathrm{K}^{-} \mathrm{N}$ potential was found in $\mathrm{K}^{-}$ nucleus scattering [8] and kaonic atoms [9]. Strong effects are expected in relativistic nucleusnucleus collisions where baryonic densities of several times the saturation density $\rho_{o}$ can be reached. Under these conditions the $\mathrm{K}^{-}$effective mass will be reduced and thus the kinematical threshold for the process $\mathrm{NN} \rightarrow \mathrm{K}^{-}+\mathrm{K}^{+}+\mathrm{NN}$ (which in free space corresponds to a kinetic beam energy of $2.5 \mathrm{GeV}$ ) will be lowered. As a consequence, the $\mathrm{K}^{-}$yield in $\mathrm{A}+\mathrm{A}$ collisions at bombarding energies below the $\mathrm{NN}$ threshold will be enhanced significantly as compared to the case without in-medium mass reduction. In contrast, the yield of $\mathrm{K}^{+}$ mesons is predicted to be decreased as the $\mathrm{K}^{+}$effective mass and thus the in-medium $\mathrm{K}^{+}$ production threshold is slightly increased [3, 10,11]. The in-medium KN potentials are also expected to affect the propagation of kaons and antikaons hence modifying their emission pattern in nucleus-nucleus collisions.

According to these considerations, the $\mathrm{K}^{-} / \mathrm{K}^{+}$ratio observed in nucleus-nucleus collisions at beam energies below the NN threshold is sensitive to the in-medium properties of kaons and antikaons. Moreover, relativistic transport calculations find distinct differences in the $\mathrm{K}^{+}$and $\mathrm{K}^{-}$spectral slopes, again due to medium effects. In particular, these calculations predict that the $\mathrm{K}^{-}$spectra fall off steeper than the $\mathrm{K}^{+}$spectra due to the decrease of the $\mathrm{K}^{-}$effective mass in the nuclear medium [12].

Recent experimental studies of kaon and antikaon production in $\mathrm{Ni}+\mathrm{Ni}$ collisions found 
a large $\mathrm{K}^{-}$yield at $1.8 \mathrm{AGeV}$ [13,14, a vanishing in-plane flow of $\mathrm{K}^{+}$mesons [15] and an enhanced out-of-plane emission of $\mathrm{K}^{+}$mesons [16]. These observations are indications for in-medium modifications of the K meson properties [10,11,17, 18].

In this Letter we report on the first comparative measurement of the $\mathrm{K}^{-}$and $\mathrm{K}^{+}$excitation functions in nucleus-nucleus collisions at subthreshold beam energies (i.e. at beam energies per nucleon below the NN threshold). We have chosen the system $\mathrm{C}+\mathrm{C}$ at bombarding energies between 0.8 and $2 \mathrm{AGeV}$. For each energy, differential cross sections for $\mathrm{K}$ meson production were obtained at various emission angles. In this light collision system, the loss of $\mathrm{K}^{-}$mesons via the strangeness exchange reaction $\mathrm{K}^{-} \mathrm{N} \rightarrow \mathrm{Y} \pi($ with $\mathrm{Y}=\Lambda, \Sigma)$ and its effect on the $\mathrm{K}^{-}$spectrum should be significantly smaller than in the $\mathrm{Ni}+\mathrm{Ni}$ system 13,14 .

The experiment was performed with the Kaon Spectrometer (KaoS) at the heavy ion synchrotron (SIS) at GSI in Darmstadt [19]. The ${ }^{12} \mathrm{C}$ beam had an intensity of about $1 \times 10^{8}$ ions per spill. The energy loss of the projectiles in the target (thickness $5 \mathrm{~mm}$ ) is less than $5 \mathrm{MeV}$ per nucleon. This magnetic spectrometer has a large acceptance in solid angle and momentum $\left(\Omega \approx 30 \mathrm{msr}, p_{\max } / p_{\min } \approx 2\right)$. The short distance of $5-6.5 \mathrm{~m}$ from target to focal plane minimizes kaon decays in flight. Particle identification and the trigger are based on separate measurements of velocity, momentum and time-of-flight. The trigger suppresses pions and protons by factors of $10^{2}$ and $10^{3}$, respectively. The background due to spurious tracks and pile-up is removed by trajectory reconstruction based on three largearea multi-wire chambers. The remaining background below the kaon mass peak $(\approx 20 \%)$ is subtracted. The loss of kaons decaying in flight is determined (and corrected) by Monte Carlo simulations using the GEANT code. The K mesons were registered at polar angles of $\Theta_{l a b}=32^{\circ}-70^{\circ}$ over a momentum range of $260<\mathrm{p}_{l a b}<1200 \mathrm{MeV} / \mathrm{c}$. The (approximate) raw numbers of detected $\mathrm{K}$ mesons are listed in Table 1.

Figure 1 shows the invariant inclusive cross section for $\mathrm{K}^{+}$(open symbols) and $\mathrm{K}^{-}$ production (full symbols) as function of the center-of-mass kinetic energy measured in $\mathrm{C}+\mathrm{C}$ collisions at $0.8,1.0,1.2,1.5,1.8$ and $2 \mathrm{AGeV}$ under different laboratory angles. The error 
bars shown are due to statistics only. An overall systematic error of $10 \%$ due to efficiency corrections and normalization procedures has to be added. The lines represent Boltzmann distributions $\mathrm{d}^{3} \sigma / \mathrm{dp}^{3} \propto \exp (-\mathrm{E} / \mathrm{T})$ fitted to the spectra (only some of the fits are shown). The resulting inverse slope parameters $\mathrm{T}$ are given in Table 1 . The T-values for the antikaons are significantly smaller than the ones for the kaons.

From the $\mathrm{K}^{+}$spectra taken at 1.0 and $1.8 \mathrm{AGeV}$ one can deduce a polar angle distribution which turns out to be forward-backward peaked. The nonisotropic contribution to the kaon yield is about $20 \%$. This value agrees with the prediction of relativistic transport calculations for $\mathrm{C}+\mathrm{C}$ collisions at 1.8 and $2.0 \mathrm{AGeV}$. Inclusive production cross sections $\sigma_{K}$ are calculated from the extrapolations in c.m. energy and angles. The kaon and antikaon spectra are parameterized by Boltzmann distributions in accordance with the data in fig.1, with the results of other measurements [13,14,20] and with the results of model calculations [12]. A nonisotropic contribution of $20 \%$ is taken into account both for $\mathrm{K}^{+}$and $\mathrm{K}^{-}$mesons although the transport calculations predict a value of $40 \%$ for the antikaons. We do not apply this correction to the data but rather include this uncertainty into the systematic error of the antikaon yields.

In Fig. 2 we present the $\mathrm{K}^{+}$and $\mathrm{K}^{-}$multiplicities per participating nucleon for $\mathrm{C}+\mathrm{C}$ collisions as a function of the energy above threshold $\left(\sqrt{s}-\sqrt{s}_{t h}\right)$ in the nucleon-nucleon $(\mathrm{NN})$ system. The values of $\sqrt{s}-\sqrt{s}_{t h}$ are calculated according to $s=\left(\mathrm{E}_{\text {pro }}+2 \mathrm{~m}_{N}\right)^{2}-\mathrm{p}_{\text {pro }}^{2}$ with $\mathrm{E}_{\text {pro }}$ and $\mathrm{p}_{\text {pro }}$ the projectile kinetic energy and momentum per nucleon and $\mathrm{m}_{N}$ the nucleon mass. The threshold energy is $\sqrt{s}_{t h}=\mathrm{m}_{K}+\mathrm{m}_{\Lambda}+\mathrm{m}_{N}=2.55 \mathrm{GeV}$ for $\mathrm{K}^{+}$production and $\sqrt{s}_{t h}=2 \mathrm{~m}_{K}+2 \mathrm{~m}_{N}=2.86 \mathrm{GeV}$ for $\mathrm{K}^{-} \mathrm{K}^{+}$pair production. $\mathrm{K}$ meson multiplicities are defined as $\mathrm{M}_{K}=\sigma_{K} / \sigma_{R}$ with $\sigma_{R}$ the geometrical cross section of the reaction which is $\sigma_{R}$ $=4 \pi\left(1.2 \mathrm{~A}^{1 / 3}\right)^{2} \mathrm{fm}^{2}=0.94 \mathrm{~b}$ for $\mathrm{C}+\mathrm{C}$. The average number of nucleons which participate in a reaction is $\left\langle\mathrm{A}_{\text {part }}>=\mathrm{A} / 2=6\right.$ for $\mathrm{C}+\mathrm{C}$ (according to a geometrical model [21]). For comparison, Fig. 2 presents also the parameterizations of the isospin averaged cross sections for $\mathrm{K}$ meson production in nucleon-nucleon collisions [22 24]. These calculations reproduce the available experimental cross sections including the most recent data measured 
at COSY close to threshold [25]. The multiplicities as shown in Fig. 2 are calculated from the elementary cross sections using $\sigma_{R}=45 \mathrm{mb}$ and $\mathrm{A}_{\text {part }}=2$ for nucleon-nucleon collisions.

The data in Fig. 2 demonstrate that the excitation functions for $\mathrm{K}^{+}$and $\mathrm{K}^{-}$production in $\mathrm{C}+\mathrm{C}$ collisions are quite similar when correcting the energy axis for the threshold energies. This similarity is also observed when the invariant differential cross sections for $\mathrm{K}^{+}$and $\mathrm{K}^{-}$ production are compared at equivalent beam energies (see Fig.1). In nucleon-nucleon (NN) collisions, however, the $\mathrm{K}^{+}$yield exceeds the $\mathrm{K}^{-}$yield by 1-2 orders of magnitude for beam energies close to threshold. The comparison of the $\mathrm{K}^{+}$and $\mathrm{K}^{-}$excitation functions for $\mathrm{C}+\mathrm{C}$ and nucleon-nucleon reactions near threshold clearly indicates that in the nuclear medium the $\mathrm{K}^{-} / \mathrm{K}^{+}$ratio is much larger than in nucleon-nucleon interactions.

In nucleus-nucleus collisions, strange mesons can also be produced in secondary processes like $\pi \mathrm{N} \rightarrow \mathrm{K}^{+} \mathrm{Y}, \Delta \mathrm{N} \rightarrow \mathrm{K}^{+} \mathrm{YN}, \pi \mathrm{N} \rightarrow \mathrm{K}^{-} \mathrm{K}^{+} \mathrm{N}, \Delta \mathrm{N} \rightarrow \mathrm{K}^{-} \mathrm{K}^{+} \mathrm{NN}$ and $\pi \mathrm{Y} \rightarrow \mathrm{K}^{-} \mathrm{N}$. According to relativistic transport models the pion and $\Delta$ induced sequential processes dominate the $\mathrm{K}^{+}$ production at bombarding energies near the kinematical threshold [11,26]. In the case of $\mathrm{K}^{-}$mesons, however, the measured yield is still about 6 times larger than what is predicted to emerge from all pion induced processes (including the strangeness exchange reaction $\left.\pi \mathrm{Y} \rightarrow \mathrm{K}^{-} \mathrm{N}\right)$. In this comparison the in-medium mass modifications are omitted but absorption is taken into account [11].

Transport calculations predict different spectral slopes for $\mathrm{K}^{-}$and $\mathrm{K}^{+}$mesons as a result of the differences in the effective masses [12,27]. Figure 3 presents the ratio of the invariant production cross sections $\left(\mathrm{K}^{-} / \mathrm{K}^{+}\right)$as a function of the c.m. kinetic energy in $\mathrm{C}+\mathrm{C}$ collisions at $1.8 \mathrm{AGeV}$. In such a representation the systematic uncertainties of the experimental data are partly compensated and the difference in slope is clearly visible. The $\mathrm{K}^{-} / \mathrm{K}^{+}$ratio steeply decreases with increasing kinetic energy of the $\mathrm{K}$ mesons. This is not a trivial observation as $\mathrm{K}^{-}$mesons with low energies are more strongly affected by absorption than $\mathrm{K}^{-}$mesons with higher energies [28]. Relativistic transport calculations predict a constant $\mathrm{K}^{-} / \mathrm{K}^{+}$ratio when in-medium mass modifications of the $\mathrm{K}$ mesons are neglected [12]. If the calculations take into account in-medium effects, both the yields and the spectral shapes of the K mesons 
are modified. In this case the $\mathrm{K}^{-}$yield at low momenta is enhanced by a factor of about 5 (e.g. at $\mathrm{E}_{c m}^{k i n}=0.1 \mathrm{GeV}$ ) mainly due to the reduced $\mathrm{K}^{-}$effective mass which drops according to $\mathrm{m}^{*}=\mathrm{m}^{0}\left(1-0.24 \rho / \rho_{0}\right)$ 12]. This effect overcompensates the absorption of $\mathrm{K}^{-}$mesons by strangeness exchange reactions $\mathrm{K}^{-} \mathrm{N} \rightarrow \mathrm{Y} \pi$ which are expected to reduce the $\mathrm{K}^{-}$yield predominantly at low kaon momenta 28].

In $\mathrm{Ni}+\mathrm{Ni}$ collisions at $1.8 \mathrm{AGeV}$, a $\mathrm{K}^{+} / \mathrm{K}^{-}$cross section ratio of $21 \pm 9$ was found [14. According to Table 1 the corresponding ratio is $39 \pm 6$ in $\mathrm{C}+\mathrm{C}$ collisions. This result is in contrast to the expectation that in the light system the absorption of $\mathrm{K}^{-}$mesons should be reduced (the $\mathrm{K}^{+}$meson cannot be absorbed in nuclear matter due to its $\bar{s}$ quark content). The observation of a decreasing $\mathrm{K}^{+} / \mathrm{K}^{-}$ratio with increasing size of the collision system may be partly caused by phase space effects: at $1.8 \mathrm{AGeV}, \mathrm{K}^{+}$production is above threshold whereas $\mathrm{K}^{-}$production is below, and the probability for subthreshold particle production via multiple collisions rises with increasing number of participants. Another reason could be the density dependence of the $\mathrm{K}^{-}$effective mass. An increasing system size corresponds to an increased average baryonic density which in turn may cause a more strongly reduction of the $\mathrm{K}^{-}$effective mass and thus a lower effective $\mathrm{K}^{-}$production threshold. The resulting relative $\mathrm{K}^{-}$yield is enhanced in the heavier system because the absorption is overcompensated by the lowered threshold.

In summary, we have measured $\mathrm{K}^{+}$and $\mathrm{K}^{-}$production in $\mathrm{C}+\mathrm{C}$ collisions at beam energies below and near the kinematical threshold. The excitation functions nearly coincide when correcting for the threshold energy. In contrast, the $\mathrm{K}^{-} / \mathrm{K}^{+}$ratio measured in $\mathrm{p}+\mathrm{p}$ collisions is about 0.01 for beam energies near threshold. The spectral slopes of the $\mathrm{K}^{-}$mesons are steeper than the ones of $\mathrm{K}^{+}$mesons at the same beam energy. Within the framework of transport calculations, both the enhanced $\mathrm{K}^{-}$yield and the steep $\mathrm{K}^{-}$spectral slope is explained by a reduced effective mass of the $\mathrm{K}^{-}$meson in the nuclear medium.

This work was supported by the German Federal Government (BMBF), by the Polish Committee of Scientific Research (Contract No. 2P03B11515) and by the GSI fund for University collaborations. 


\section{REFERENCES}

[1] D.B.Kaplan and A.E.Nelson, Phys.Lett. B 175 (1986) 57

[2] G.E.Brown, C.H.Lee, M.Rho and V.Thorsson, Nucl.Phys. A 567 (1994) 937

[3] J.Schaffner-Bielich, J.Bondorf, I.Mishustin, Nucl. Phys. A 625 (1997) 325

[4] T.Waas, N.Kaiser and W.Weise, Phys.Lett. B 379 (1996) 34

[5] M.Lutz, Phys.Lett. B 426 (1998) 12

[6] G.E.Brown and H.A.Bethe, Astrophys.Jour. 423 (1994) 659

[7] G.Q.Li, C.H.Lee and G.E.Brown, Phys.Rev.Lett. 79 (1997) 5214

[8] E.Friedman and A.Gal, Phys.Lett. B 308 (1993) 6

[9] E.Friedman et al., Phys.Rev. C 55 (1997) 1304

[10] G.Q.Li, C.M.Ko, X.S.Fang, Phys.Lett. B 329 (1994) 149

[11] W.Cassing et al., Nucl.Phys. A 614 (1997) 415

[12] E.Bratkovskaya, W.Cassing, U.Mosel, Phys.Lett. B 424 (1998) 244

[13] A.Schröter et al., Z.Phys. A 350 (1994) 101

[14] R.Barth et al., Phys.Rev.Lett. 78 (1997) 4007

[15] J.Ritman et al., Z.Phys. A 352 (1995) 355

[16] Y.Shin et al., Phys.Rev.Lett. 81 (1998) 1576

[17] G.Q.Li et al.,Phys.Rev.Lett. 74 (1995) 235 and Phys.Lett. B 381 (1996) 17

[18] G.Q.Li, C.M.Ko and G.E.Brown, Phys.Lett. B 381 (1996) 17

[19] P.Senger et al., Nucl. Instr. Meth. A 327 (1993) 393

[20] W.Ahner et al., Phys. Lett. B 393 (1997) 31 
[21] J.Hüfner und J.Knoll, Nucl.Phys. A 290 (1977) 460

[22] A.Sibirtsev, Phys.Lett. B 359 (1995) 29

[23] E.Bratkovskaya, W.Cassing and U.Mosel, Nucl.Phys. A 622 (1997) 593

[24] A.Sibirtsev, W.Cassing and C.M.Ko, Z.Phys. A 358 (1997) 101

[25] J.Balewski et al., Phys.Lett. B 388 (1996) 859 and Phys.Lett. B in print

[26] C.Fuchs et al., Phys.Rev. C 56 (1997) R606

[27] W.Cassing and E.Bratkovskaya, Phys.Rep. (1998) in print

[28] C.B.Dover and G.E.Walker, Phys.Rep.89 (1982) 1 


\section{TABLES}

Table 1: Sample sizes, inverse slope parameters and inclusive production cross sections for kaons and antikaons in $\mathrm{C}+\mathrm{C}$ collisions. The values for $\mathrm{T}$ and $\sigma$ are determined by fitting a Boltzmann distribution $\mathrm{d}^{3} \sigma / \mathrm{dp}^{3} \propto \exp (-\mathrm{E} / \mathrm{T})$ to the data. In the extrapolation to full phase space the nonisotropic angular distribution is taken into account (see text). The quoted errors on the cross sections include systematic effects.

\begin{tabular}{|c|c|c|c|c|}
\hline $\begin{array}{c}\text { beam energy } \\
(\mathrm{AGeV})\end{array}$ & $\Theta_{l a b}$ & $\mathrm{~N}\left(\mathrm{~K}^{+}\right)$ & $\begin{array}{l}\mathrm{T}\left(\mathrm{K}^{+}\right) \\
(\mathrm{MeV})\end{array}$ & $\begin{array}{c}\sigma\left(\mathrm{K}^{+}\right) \\
(\mathrm{mb})\end{array}$ \\
\hline 0.8 & $44^{\circ}$ & 740 & $54 \pm 3$ & $0.015 \pm 0.003$ \\
\hline 1.0 & $44^{\circ}, 54^{\circ}$ & 1200 & $58 \pm 6$ & $0.1 \pm 0.02$ \\
\hline 1.2 & $40^{\circ}$ & 2400 & $64 \pm 4$ & $0.3 \pm 0.05$ \\
\hline 1.5 & $32^{\circ}, 48^{\circ}$ & 7000 & $74 \pm 5$ & $1.3 \pm 0.2$ \\
\hline 1.8 & $32^{\circ}, 40^{\circ}, 48^{\circ}, 60^{\circ}$ & 17000 & $75 \pm 5$ & $3.0 \pm 0.3$ \\
\hline 2.0 & $32^{\circ}, 40^{\circ}$ & 11000 & $78 \pm 5$ & $5.0 \pm 0.5$ \\
\hline $\begin{array}{c}\text { beam energy } \\
(\mathrm{AGeV})\end{array}$ & $\Theta_{l a b}$ & $\mathrm{~N}\left(\mathrm{~K}^{-}\right)$ & $\begin{array}{l}\mathrm{T}\left(\mathrm{K}^{-}\right) \\
(\mathrm{MeV})\end{array}$ & $\begin{array}{c}\sigma\left(\mathrm{K}^{-}\right) \\
(\mathrm{mb})\end{array}$ \\
\hline 1.5 & $40^{\circ}$ & 50 & $40 \pm 15$ & $0.016 \pm 0.006$ \\
\hline 1.8 & $40^{\circ}, 60^{\circ}$ & 2000 & $55 \pm 6$ & $0.076 \pm 0.02$ \\
\hline 2.0 & $40^{\circ}$ & 370 & $55 \pm 7$ & $0.19 \pm 0.06$ \\
\hline
\end{tabular}


Figure 1: Inclusive invariant production cross-sections of $\mathrm{K}^{+}$mesons (open symbols) and $\mathrm{K}^{-}$mesons (full symbols) measured in $\mathrm{C}+\mathrm{C}$ collisions at beam energies of $2,1.8,1.5,1.2$, 1.0 and $0.8 \mathrm{AGeV}$ (from top to bottom) under different laboratory angles (as indicated). The solid lines correspond to Boltzmann distributions fitted to some of the spectra (see text and Table 1).

Figure 2:

Kaon and antikaon multiplicity per participating nucleon as a function of the Q-value for $\mathrm{C}+\mathrm{C}$ collisions (open squares: $\mathrm{K}^{+}$, full dots: $\mathrm{K}^{-}$) and nucleon-nucleon collisions. The error bars include systematic effects. The lines correspond to parameterizations of the isospin averaged cross sections for $\mathrm{K}$ meson production measured in proton-proton collisions (full line: $\mathrm{K}^{+}$, dashed line: $\mathrm{K}^{-}$) [22 24].

Figure 3:

$\mathrm{K}^{-} / \mathrm{K}^{+}$ratio as a function of the center-of-mass kinetic energy measured in $\mathrm{C}+\mathrm{C}$ collisions at $1.8 \mathrm{AGeV}$ and at $\Theta_{l a b}=40^{\circ}$. 


\section{FIGURES}

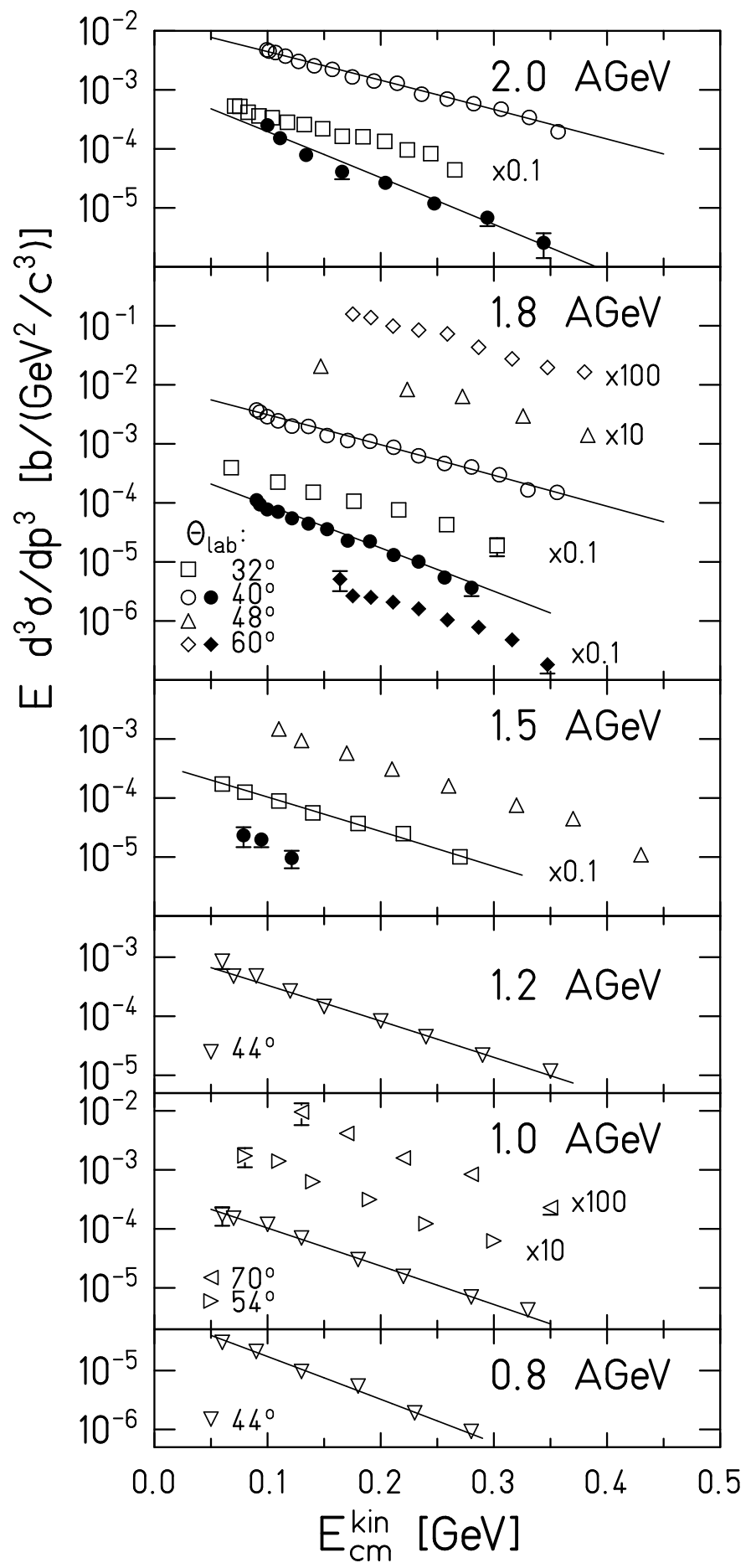


FIG. 1.

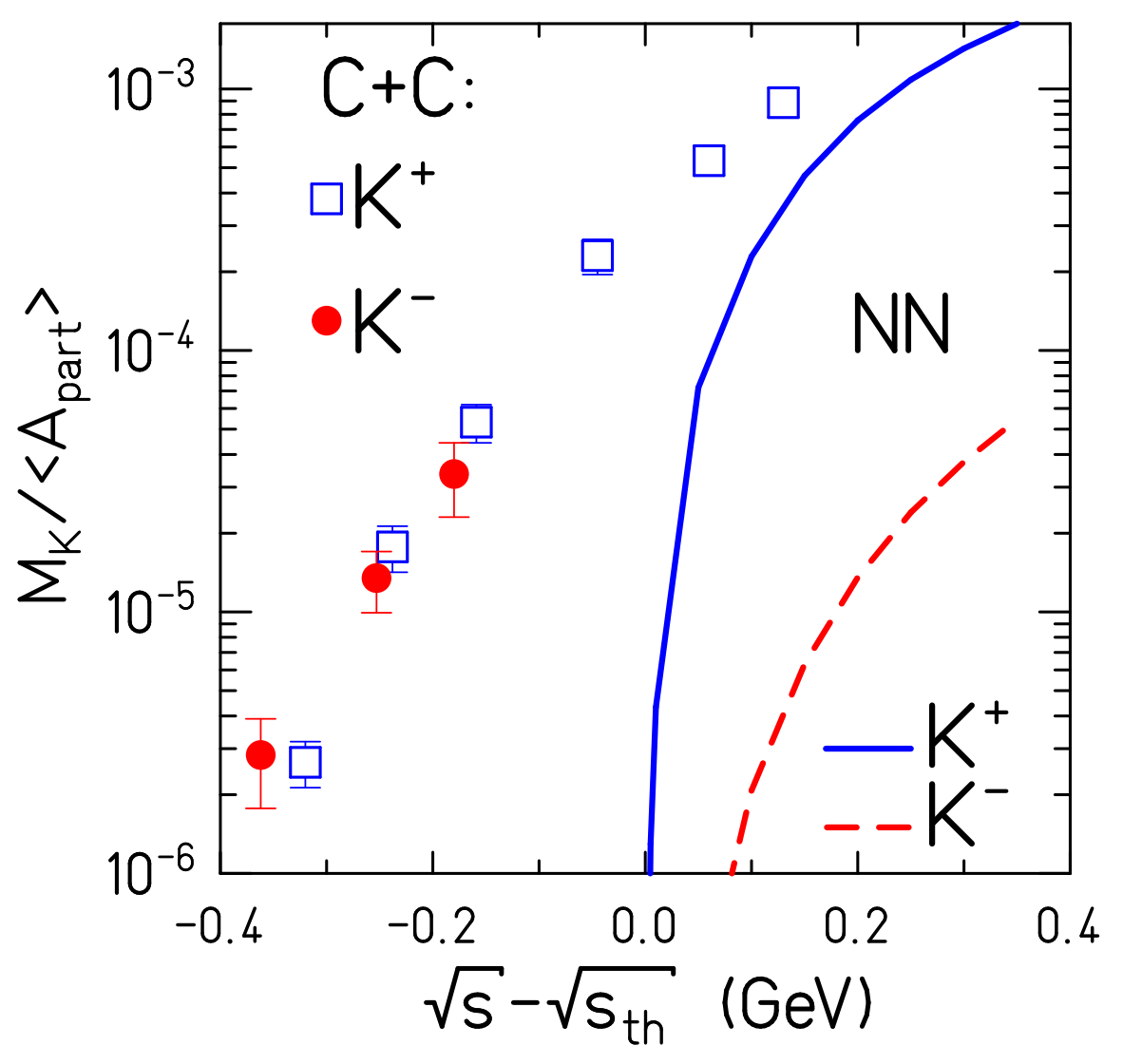

FIG. 2. 


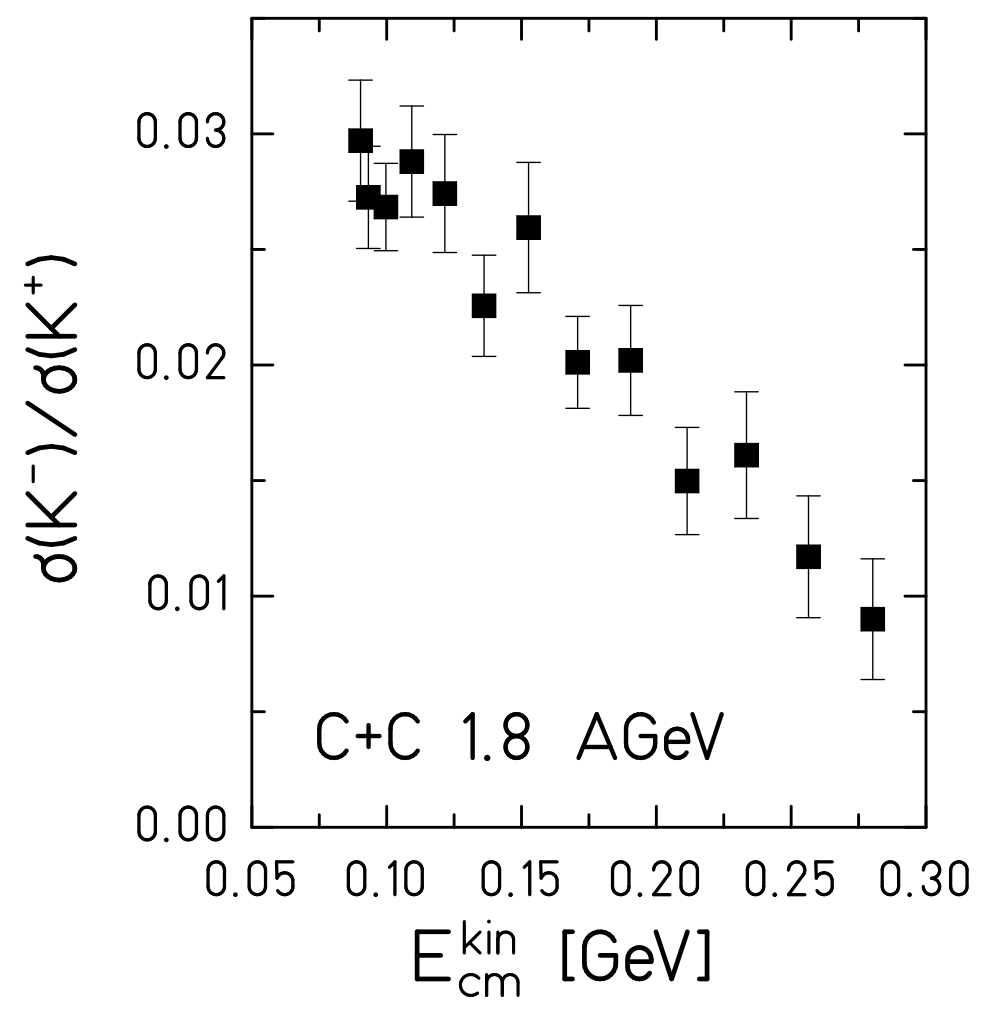

FIG. 3. 